Ann. Abeille, I96r, 4 (2), I59-I70.

\title{
TRAVAUX DES STATIONS DE BURES-SUR-YVETTE ET DE MONTFAVET EN 1960
}

\author{
R. CHAUVIN.
}

\section{LES SUB'TANCES DE FAMILIARISATION DANS LA RUCHE.}

Chauvin a procédé à la récapitulation de ces substances très répandues dans la ruche et dont le rôle paraît très complexe. On peut les englober dans le groupe des " phérormones " de BUTENANDT et KARLSON, substances émises par un individu et reçues par un autre de la même espèce, chez lequel elles induisent des réactions déterminées. Mais une telle définition est trop vague et exige des subdivisions. On ne peut mettre côte à côte des facteurs dont l'effet est opposé : c'est pourquoi ChauviN propose de distinguer des épagines qui, déposées par les ouvrières sur divers supports, suppriment leurs réactions de fuite devant un objet qu'elles n'ont jamais rencontré. On en connaît jusqu'à présent sept espèces, après les travaux de VuillaUme et LEComTe. Les répulsines qui induisent au contraire la réaction d'évitement sont au nombre de six et VUILLAUME et LECoMTE les ont également étudiées. Enfin les allectines ou substances de butinage existent probablement dans le nectar et sûrement dans le pollen.

\section{IA PHÉRORMONE DES REINES.}

Quant à la phérormone proprement dite, elle a fait l'objet des travaux de Mille PaIN, depuis de nombreuses années. Cette substance, émise par la reine, attire fortement les ouvrières, les empêche de construire des cellules royales, inhibe le développement de leurs ovaires, et a sans doute bien d'autres effets que nous ne soupçonnons guère (par exemple elle accélère les activités de construction, voir plus loin). C'est sur l'attractivité de ce corps que Mile PAIN a fait porter la plus grande partie de ses efforts. Lorsque BuTLER a étudié l'action de la substance royale sur la construction des cellules royales, le test qu'il employait ne lui permettait pas de mettre en évidence le pouvoir d'attraction de cette substance. Or, elle comprend plusieurs constituants : l'un d'eux, l'acide céto-9- décène-2; transoïque, est responsable de l'inhibition des cellules royales; mais il est complètement inattractif Chauvin propose de le désigner sous le nom de phérormone 1 . Pour qu'il devienne attractif, il faut qu'il soit mélangé à une autre fraction toute différente, plus volatile, et qui se compose elle-même de deux ou trois corps (phérormone II). Il faut insister sur le fait que c'est le mélange phérormone I + phérormone II qui est attractif, alors qu'aucune des deux fractions ne l'est séparément. Les deux phérormones se rencontrent dans les glandes mandibulaires des reines à un taux variable qui dépend de l'âge de la reine et du fait qu'on l'a mise ou non en présence des ouvrières. La phérormone peut d'ailJeurs s'extraire du corps des ouvrières qui ont fréquenté la reine, mais non du corps des orphelines (Barbier et et Pain ; Pain, Hügel et Barbier ; Pain et Barbier). Darchen trouve d'autre part que la reine exerce une grosse influence sur le comportement de construction des ouvrières ; même 
des parties de son corps, comme la tête ( et la tête surtout) suffisent à accélérer la construction. Il devient possible alors d'instituer une autre série de tests biologiques, qui mesureraient sans doute de nouveaux effets de la phérormone.

\section{LA FORMATION DES GUFS DANS LES OVAIRES DES OUVRIÈRES ET L'EFFET DE GROUPE.}

PaIN a mis en évidence un très curieux phénomène chez les ouvrières orphelines. Reprenant les observations de Hess, suivant laquelle chez les ouvrières isolées les ovaires restent atrophiés pendant un temps très long, PAIN montre que le groupement des abeilles par 2 suffit à accélérer notablement l'apparition du premier ouf dans les ovarioles; et mieux encore le groupement par 3 ou davantage. Mais dans le cas du groupement par 2, on voit de plus s'établir une sorte de "dominance " en ce sens que les ovarioles de l'une des deux abeilles se développent presque toujours plus que ceux de l'autre. Il n'est pas impossible qu'il ne faille l'attribuer à une inégalité des échanges de nourriture entre les deux partenaires l'une jouant surtout le rôle de donneuse et l'autre de receveuse.

\section{LA POLYGYNIE EXPÉRIMENTALE.}

Tous les auteurs ont remarqué depuis fort longtemps les difficultés que l'on rencontre dans les opérations chirurgicales sur les ouvrières. La mortalité est en effet très élevée et il est rare que les opérées survivent plus de quelques jours. Mais les reines semblent plus résistantes et DARCHEN a réussi à couper le dard des reines sans nuire à la ponte ; d'où il s'ensuit que cet organe ne doit pas jouer le rôle qu'on lui supposait dans la guidage des œufs, ou tout au moins que ce rôle n'est pas indispensable. Il est plus curieux de noter que passée une première phase de combat, la cohabitation devient possible pour ces reines à dard coupé. On peut même, à l'aide de procédés simples, amener les reines à pondre ensemble dans une ruche normale ; ces recherches sont en plein développement, mais pourraient ouvrir à l'apiculture des possibilités insoupçonnées.

\section{IE COMPORTEMENT.}

Vuilladme et Nadlleau se sont attachés à préciser les remaniements des cupules de cire utilisées pour l'élevage des reines et qui n'ont pas été acceptées par les ouvrières. Toutes une série de modifications régulières sont décrites, qui se caractérisent notamment par le passage quasi obligatorre au stade de cellules arrondies.

ROPARTZ note que le photopréférendum des abeilles dépend essentiellement des interactions sociales. Lorsque la grappe est bien formée, les abeilles ne se soucient presque pas de la lumière.

Naulleau étudie les transpositions des larves dans les cellules de leur propre caste ou d'autres castes. Cette transposition entraîne l'élimination de presque toutes les larves à moins qu'on n'opère sur une grande quantité à la fois. La sensibilité des nourrices aux transpositions est moins grande aans les colonies orphelines que dans celles qui sont pourvues d'une reine. Dans ce dernier cas, phénomène qui paraît difficile à interpréter, même le simple déplacement d'une larve dans sa propre cellule, où le crochet de l'expérimentateur s'est contenté de la repousser légèrement, peut entraîner son élimination. Ajoutons d'ailleurs que Nadlleau a pu constater le transport spontané par les Juvrières de certaines larves d'une cellule à l'autre. Les larves de reine transférées par l'expérimentateur dans des cellules d'ouvrières donnent des formes intermédiaires. Les larves de reine translérées dans les cellules de mâles sont mortes jusqu'à présent.

LeComte a pu améliorer les méthodes d'étude du butinage en employant les radioisotopes. Le sirop de sucre radioactif, en période de grande miellée, n'est pas l'objet d'échanges aussi intenses que l'ont écrit Nixon et RibBands. Divers groupes de travail paraissent alors se spécialiser sur différentes espèces florales, et n'ont guère de contacts entre eux. D'autre part la dispersion des butineuses est très hétérogène : ainsi, de deux champs d'espèces florales différentes placés côte à côte, l'un peut 
être visité et l'autre non ; de deux champs de même espèce situés à proximité de la ruche, mais à des emplacements différents, l'un peut être assidûment exploité et l'autre non. L'effet perturbateur du relief est considérable, ainsi que l'effet répulsif de grandes étendues stériles.

\section{LES PRODUITS DE LA RUCHE.}

LAviE a publié cette année sa thèse sur les substances antibactériennes dans la ruche ; c'est un travail trop considérable pour qu'il soit possible de le résumer ici. Notons toutefois que des substances antibactériennes ont été trouvées dans le miel, le pollen, la gelée royale, la cire, la propolis et le corps même des abeilles. Leur activité a été comparée sur un grand nombre de souches bactériennes. Certaines de ces substances sont également inhibitrices de la germination (surtout les extraits de propolis et de miel) (GoNnET et LAVIE). La propolis est de plus fortement antifongique. D'autre part, l'antibiotique du miel qui serait, d'après les Allemands, aisément détruit par la chaleur a été trouvé au contraire relativement stable par GoNNET et LAVIE. Tout paraît dépendre d'ailleurs du temps de chauffage, de la nature du miel et surtout de son $\mathrm{pH}$.

\section{TECHNOLOGIE}

A signaler une revue de FrESNAYE sur la protection du bois des ruches contre les intempéries une exposition de deux ans à l'air libre permet déjà d'éliminer certains types de peintures et revêtement.

\section{REVUES GÉNÉRALES}

Chauvin a fait la revue des travaux les plus importants sur la biologie de l'abeille parus de 1956 à I960. Maurizio et Louveaux ont étudié avec beaucoup de détails quelques pollens de grande im. portance apicole. DARCHEN a participé à la rédaction de l'ouvrage apicole allemand "Biene und Bienenzucht ".

\section{VISITES, RÉUNIONS, etc...}

Ont accompli des stages à la station pendant 1960 : MM. LENSKY d'Israël, JoYAPPA de l'Inde, RiHAR et Ilesic de Yougoslavie, Romero FABre d'Espagne. Nous avons reçu la visite de M. Avidov, doyen de la Faculté d'Agriculture de Rehovot (Israël) et du professeur Syunro Utidd (Japon). M. KoenLer (Allemagne) a présenté à la Station un exposé de ses travaux.

\section{NOTES PARUES AU COURS DE L'ANNÉE.}

(I) Barbier M., Pain J. Etude de la sécrétion des glandes mandibulaires des reines et des ouvrières d'abeille. C. R. Acad. Sc., 250, 3740-2.

(2) Chauvin R. Progrès récents dans la Biologie de l'Abeille. Ann. Ab., IV, 5-39.

(3) Chauvin R. Les substances actives sur le comportement à l'intérieur de la ruche. Ann. Ab., IV, I $85-97$.

(4) Darchen R. Die Bienengemeinschaft. (Sonderdruck aus "Biene und Bienenucht ", Ehrenwirth Verlag, Mïnchen, I960, 53-67).

(5) Darchen R. L'ablation du dard des reines et des ouvrières d'Apis mellifica. C. R. Acad. Sc., 250, $203-4$.

(6) DARChen R. Le polygynisme expérimental dans les colonies d'Apis $\boldsymbol{m}$ ellifica. Ibid, 250, 934-6.

(7) Darchen R. Les régulations neurohormonales et l'instinct constructeur des ouvrières d'Apis mellifica. Ann. Ab., IV, 329-33.

(8) Fresnaye J. Deux années d'expérimentation sur la protection du bois des ruches. Ann. Ab., IV, $85-98$. (9) Gonnet M., Lavie P. Influence du chauffage sur le facteur antibiotique présent dans les miels.;
Ann. $A b$. IV, 349-64.

(io) Gonnet M., Lavie P. Action antigerminative des produits de la ruche d'abeilles (Apis mellifica L.) sur les graines et les tubercules. $C$. $R$. Acad. Sc., 250, 6r2-14. 
(1 1) LAviE P. Les substances antibactériennes dans la colonie d'abeilles Apis mellifica L. (Thèse Université Paris, parue dans les Annales de l'Abeille, rgo pp.

(12) Leconte J. Observations sur le comportement des Abeilles butineuses. Ann. Ab., IV, 3I 7-27.

(i3) Maurizio A., Louveaux J. Pollen des plantes mellifères d'Europe. Pollen et Spores, 11, i $50-82$.

(I4) Nalileau G. Les transpositions de larves de différentes castes d'A pis mellifica dans les cellules d'autres castes. Etude des types intermédiaires entre reines et ouvrières. Ann. $A b$., IV, 65-83.

(15) Pain J., Huged M. F., Barbier M. Sur les constituants des mélanges attractifs des glandes mandibulaires des reines d'Abeille (Apis mellifica L.) à difiérents stades de leur vie. C.R. Acad. Sc., 250, 1046-8. (16) Pain J., Barbier M. Mise en évidence d'une substance attractive extraite du corps d'ouvrières d'A beilles non orphelines. Ibid., I I 26-7.

(г 7) Pain J. De l'influence du nombre des Abeilles encagées sur la formation des œufs dans les ovaires de l'ouvrière. Ibid., 26, 29-3I.

(r8) Ropartz P. Sur les réactions des Abeilles à la lumière. Ann. Ab., IV, 335-8.

(ig) Vulladum M., Naullatiu G. Constructions de cellules rondes et de cellules irrégulières chez Apis mellifica. Ann. Ab., IV, $45-63$. 